\title{
Bacterial Filtration Using Carbon Nanotube/Antibiotic Buckypaper Membranes
}

\author{
Luke J. Sweetman, ${ }^{1}$ Leighton J. Alcock, ${ }^{1}$ Jason D. McArthur, ${ }^{2}$ Elise M. Stewart, ${ }^{3}$ \\ Gerry Triani, ${ }^{4}$ Marc in het Panhuis, ${ }^{1,3}$ and Stephen F. Ralph ${ }^{1}$ \\ ${ }^{1}$ Soft Materials Group, School of Chemistry, University of Wollongong, Wollongong, NSW 2522, Australia \\ ${ }^{2}$ Illawarra Health and Medical Research Institute, University of Wollongong, Wollongong, NSW 2522, Australia \\ ${ }^{3}$ Intelligent Polymer Research Institute, ARC Centre for Excellence for Electromaterials Science, AIIM Facility, \\ University of Wollongong, Wollongong, NSW 2522, Australia \\ ${ }^{4}$ Institute of Materials Engineering, Australian Nuclear Science and Technology Organisation, Lucas Heights, NSW 2234, Australia
}

Correspondence should be addressed to Stephen F. Ralph; sralph@uow.edu.au

Received 7 November 2012; Revised 28 January 2013; Accepted 8 February 2013

Academic Editor: Yanbao Zhao

Copyright (C) 2013 Luke J. Sweetman et al. This is an open access article distributed under the Creative Commons Attribution License, which permits unrestricted use, distribution, and reproduction in any medium, provided the original work is properly cited.

\begin{abstract}
The preparation of free-standing carbon nanotube "buckypaper" (BP) membranes consisting of either single-walled carbon nanotubes (SWNTs) or multi-walled carbon nanotubes (MWNTs), and the antibiotic ciprofloxacin (cipro), is reported. The electrical, mechanical and morphological properties of these membranes have been characterised and are compared to those of the corresponding buckypaper membranes containing the surfactant Triton X-100 (Trix). Analysis of scanning electron microscopic images of the surfaces of SWNT/cipro and SWNT/Trix (Trix = Triton X-100) buckypapers revealed that the diameter of their surface pores was significantly smaller than that of the corresponding materials prepared using MWNTs. Similarly, the average internal pore diameter of both SWNT buckypapers was found to be smaller than that of their MWNT counterparts, after analysis of binding isotherms derived from nitrogen adsorption/desorption measurements performed on the materials. All four buckypaper membranes examined were found to be $>99 \%$ effective for removing Escherichia coli (E. coli) from aqueous suspensions. However, buckypapers containing ciprofloxacin outperformed their counterparts containing the surfactant. Both MWNT buckypapers were more effective at preventing passage of $E$. coli than their analogues containing SWNTs, while fluorescence microscopic examination of stained membrane surfaces demonstrated that buckypapers composed of SWNTs had greater bactericidal properties.
\end{abstract}

\section{Introduction}

Guarding water supplies against contamination from pathogenic organisms remain one of the most important challenges facing society $[1,2]$. While filtration methods are effective for removing microbial contaminants, the susceptibility of nanofiltration and reverse osmosis membranes to fouling necessitates the use of additional disinfection processes to ensure that pathogenic organisms do not enter water supplies $[3,4]$. A further weakness of current nanofiltration membranes is they lack analyte specificity $[4,5]$. Consequently, there is an ongoing need to develop new membrane materials $[5,6]$.

Membranes composed of aligned arrays of carbon nanotubes (CNTs) have shown very high permeabilities towards water and gases $[7,8]$, as well as the ability to discriminate between molecules or nanoparticles on the basis of differences in their sizes [7]. They have also proven effective for removing bacteria and virus particles from water samples [9], while several other studies have shown that CNTs have antimicrobial properties $[10,11]$. This effect was more pronounced with single-walled carbon nanotubes (SWNTs) than multi-walled carbon nanotubes (MWNTs), which was attributed in part to the greater ability of the former to physically penetrate bacterial cell walls [11]. Inspired in part by these results, Brady-Estévez and coworkers studied the antibacterial properties of CNT membranes and composite materials $[12,13]$. One of their first studies involved buckypapers made from SWNT dispersions prepared in dimethylsulfoxide [12]. These materials were prepared without 
the assistance of a dispersant molecule and proved highly effective at removing E. coli from aqueous solutions. Metabolic and viability assays performed on the surface of the BPs after they had been used for filtration experiments showed that the majority of the bacteria that had been retained were metabolically inactive and had compromised membranes. These buckypapers also proved effective at removing viral particles from solution, highlighting their potential utility for treatment of contaminated water supplies.

We recently used macrocyclic ligands to assist in the preparation of aqueous dispersions of SWNTs and buckypapers that retained the ligand molecules [14]. Comparison to buckypapers prepared using Triton X-100 (Trix) as the dispersant revealed that incorporation of the macrocycles sometimes resulted in dramatic changes to the physical and morphological properties of the membranes, as well as their permeability towards water. We therefore considered that it might also be possible to use antibiotics with appropriate structural features to form CNT dispersions and buckypapers which retain antibiotic molecules and consequently display enhanced bactericidal activity. Further support for this proposal was provided by studies which showed that CNTs can remove antibiotics from aquatic environments by an adsorption mechanism $[15,16]$. However, to date the only studies that have used antibiotics to disperse CNTs focussed on the preparation of modified electrodes containing thin films composed of MWNTs and the antibiotic ciprofloxacin (cipro; Figure 1) [17-19].

In this paper we report on the ability of free-standing SWNT and MWNT buckypapers containing ciprofloxacin to filter solutions containing Escherichia coli (E. coli) and the bactericidal properties of the buckypapers. In addition, the physical and morphological properties of MWNT/cipro and SWNT/cipro buckypapers are compared to each other and to those of the corresponding membranes containing the surfactant Trix, which has no significant antibacterial activity.

\section{Experimental}

2.1. Reagents. All chemical reagents were used as received from suppliers, without any further purification. SWNTs produced by the HiPco process were obtained from Unidym (Lot no. P2150), while thin MWNTs prepared by a chemical vapour deposition method were obtained from Nanocyl (Lot no. 081010). Ciprofloxacin hydrochloride was purchased from MP Biomedicals LLC.

2.2. Preparation of Dispersions. All dispersions were prepared in Milli-Q water $(18.2 \Omega \mathrm{Mcm})$ using a SWNT or MWNT concentration of $0.1 \%(\mathrm{w} / \mathrm{v})$, and either Trix or ciprofloxacin present at a concentration of $1.0 \%(\mathrm{w} / \mathrm{v})$. In order to facilitate formation of dispersions, a high energy $(400 \mathrm{~W})$ sonication horn (probe diameter $=10 \mathrm{~mm}$; Branson 450, Ultrasonics) was used with the following parameters: amplitude $=30 \%$; pulse duration $=0.5 \mathrm{~s}$; pulse delay $=0.5 \mathrm{~s}$ ). For a typical experiment $15 \mathrm{mg}$ of CNTs were dispersed in $15 \mathrm{~mL}$ of dispersant solution. During sonication the reaction vial was placed inside an ice/water bath to minimize increases in

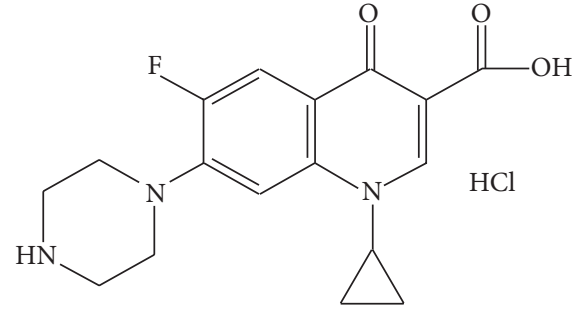

FIGURE 1: Molecular structure of ciprofloxacin hydrogen chloride.

temperature. A sonication time of $30 \mathrm{~min}$ was employed to prepare all CNT dispersions used for synthesising the buckypapers described in this paper.

2.3. Preparation of Buckypapers. In order to produce a small, circular buckypaper (approximate diameter $47 \mathrm{~mm}$ ) two dispersions, prepared as described previously, were combined and added to a further $50 \mathrm{~mL}$ of dispersant solution (either $1.0 \%(\mathrm{w} / \mathrm{v})$ Trix or cipro) and then subjected to treatment in a conventional ultrasonic bath (Unisonics; $50 \mathrm{~Hz}, 150 \mathrm{~W}$ ) for $3 \mathrm{~min}$. The solution was then diluted to a total volume of $250 \mathrm{~mL}$ using Milli-Q water and filtered under vacuum through a polytetrafluoroethylene (PTFE) membrane filter ( $5 \mu \mathrm{m}$ diameter pore size; Millipore) housed in an Aldrich glass filtration unit, by using a Vacuubrand CVC2 pump to apply a vacuum between 30 and 50 mbar.

2.4. Characterisation Techniques. Absorption spectra (400$1000 \mathrm{~nm}$ ) of all dispersions were obtained using a Cary 500 UV-vis-NIR spectrophotometer and quartz cuvettes. The dispersions were first diluted with Milli-Q water to ensure that the measured absorbances were within the optimal range of the instrument.

The surface morphology of buckypapers was examined using a JEOL JSM-7500FA FESEM. Prior to analysis, the buckypapers were cut into small strips and mounted on a small, conductive stub using carbon tape. The samples exhibited sufficient electrical conductivity to be imaged without prior sputter coating. Images obtained by scanning electron microscopy (SEM) were analysed using Image Pro Plus software to obtain quantitative information concerning the average diameter of surface pores. The average surface pore diameters of the buckypapers reported in Table 1 were determined using a single buckypaper sample. Energy Dispersive X-ray (EDX) spectroscopic analysis of the surface of buckypapers was performed concurrently to obtain information about the identity of elements present.

The contact angles of buckypapers were measured using the sessile drop method and a Data Physics SCA20 goniometer fitted with a digital camera. The contact angles of $2 \mu \mathrm{L}$ Milli-Q water droplets on the surfaces of the buckypapers were calculated using the accompanying Data Physics software (SCA20.1). The mean contact angle was calculated using measurements performed on a minimum of five water droplets. 
TABLE 1: Morphological properties of buckypapers.

\begin{tabular}{lccccc}
\hline Buckypaper & $D_{\mathrm{SEM}}{ }^{\mathrm{a}}(\mathrm{nm})$ & $A_{\mathrm{BET}}{ }^{\mathrm{b}}\left(\mathrm{m}^{2} / \mathrm{g}\right)$ & $d_{\mathrm{BET}}{ }^{\mathrm{c}, \mathrm{d}}(\mathrm{nm})$ & $D_{\text {bun }}^{\mathrm{d}, \mathrm{e}}(\mathrm{nm})$ & ${\text { Interbundle pore volume }(\%)^{\S}}^{\S}$ \\
\hline SWNT/Trix & $23 \pm 7$ & $790 \pm 4$ & $4 \pm 0.4$ & $3.4 \pm 0.1$ & $84 \pm 5$ \\
SWNT/cipro & $29 \pm 17$ & $360 \pm 1$ & $7 \pm 0.8$ & $7.5 \pm 0.1$ & $95 \pm 6$ \\
MWNT/Trix & $80 \pm 20$ & $300 \pm 1$ & $24 \pm 1$ & $8.8 \pm 0.2$ & $91 \pm 5$ \\
MWNT/cipro & $70 \pm 20$ & $250 \pm 1$ & $26 \pm 2$ & $10 \pm 1$ & $\mathrm{f}$ \\
\hline
\end{tabular}

${ }^{\mathrm{a}} D_{\mathrm{SEM}}$ : surface pore diameter derived by Image Analysis of SEM micrographs. ${ }^{\mathrm{b}} A_{\mathrm{BET}}$ : buckypaper surface area obtained through Brunauer, Emmett and Teller (BET) analysis of isotherms derived from nitrogen adsorption/desorption measurements [20]. ${ }^{\mathrm{c}}$ Average internal pore diameter. ${ }^{\mathrm{d}}$ Obtained through application of Horvath-Kawazoe (HK) and Barrett, Joyner and Halenda (BJH) methods to isotherms derived from nitrogen adsorption/desorption measurements [21, 22]. ${ }^{\mathrm{e}}$ Average nanotube bundle diameter. ${ }^{\mathrm{f}}$ Insufficient data to enable calculation of this value. ${ }^{\mathrm{g}}$ Data taken from [14].

The electrical conductivity of buckypapers was determined using a standard two-point probe method [23]. Details of the procedure employed to measure sample conductivities were described by us previously [14]. Measurements were performed on three separate strips of membrane for each type of buckypaper, with the average values reported in Table 2 . The mechanical properties of buckypapers were examined using a Shimadzu EZ-S universal testing device and buckypaper samples cut into small rectangular strips measuring $15 \mathrm{~mm} \times 3 \mathrm{~mm}$. The latter were stretched using a $10 \mathrm{~N}$ load cell at a strain rate of $1 \mathrm{~mm} \mathrm{~min}^{-1}$ until sample failure. The tensile strength was determined as the maximum stress measured, while the ductility was the percentage elongation at breaking point. The Young's modulus and sample toughness were also determined. Values for each of the previously mentioned mechanical properties are reported in Table 2 for each of the buckypapers examined and are the average of results obtained using three different strips of membrane.

Nitrogen adsorption/desorption isotherms were obtained for each type of buckypaper using a surface area analyzer (ASAP 2010 or ASAP 2020, Micromeretics) operating at $77 \mathrm{~K}$ and a single sample of each type of membrane. Prior to analysis, residual gas trapped within samples was removed under vacuum at $200^{\circ} \mathrm{C}$. The resulting isotherms were analysed using the Horvath-Kawazoe (HK) and Barrett, Joyner and Halenda $(\mathrm{BJH})$ methods to determine the distribution of small and large pores, respectively [21, 22]. In addition, multipoint Brunauer, Emmett and Teller (BET) analysis of the isotherms was used to calculate the specific surface areas of the samples [20].

2.5. Bacterial Filtration and Imaging Experiments. Escherichia coli JM109 was selected as the model bacterium throughout the course of this study. A single colony of $E$. coli was inoculated into $5 \mathrm{~mL}$ of Luria-Bertani (LB) broth and grown at $37^{\circ} \mathrm{C}$ for $16 \mathrm{~h}$ with shaking at $200 \mathrm{rpm}$. The overnight culture $(1 \mathrm{~mL})$ was used to inoculate $20 \mathrm{~mL}$ of prewarmed $\mathrm{LB}$ broth, which was subsequently incubated at $37^{\circ} \mathrm{C}$ with shaking until an $\mathrm{OD}_{600}$ of 0.5 (midexponential growth phase) was obtained. For filtration experiments, $1 \mathrm{~mL}$ of freshly prepared cells was suspended in $50 \mathrm{~mL}$ sterile saline solution $(0.9 \%(\mathrm{w} / \mathrm{v}) \mathrm{NaCl})$ giving a final cell concentration of c.a. $10^{7} \mathrm{~mL}^{-1}$. Prior to testing, the buckypaper membranes were sterilised by soaking in $70 \%$ ethanol and thoroughly washed with sterile saline to remove any remaining solution, and the glass filter holder and flask to be used for the filtration process were sterilized using an autoclave. Bacterial suspensions were filtered through dry buckypapers using a vacuum of approximately $200-300 \mathrm{mbar}$ at room temperature $\left(21^{\circ} \mathrm{C}\right)$. To determine the extent of removal of E. coli, a dilution series was prepared from the filtrate by plating onto LB agar and incubating overnight at $37^{\circ} \mathrm{C}$. The numbers of colonies present after this period of time were then counted by direct visual inspection and converted to $\log$ removal values. Table 3 shows the average log removal values for each buckypaper after performing three separate experiments.

The viability of $E$. coli on buckypaper membranes was examined using a live/dead assay performed in accordance with the procedure developed by Brady-Estévez et al. [12]. Immediately after filtration experiments, buckypapers were either stained with propidium iodide (PI) followed by counter staining with SYTO-16 or stained with PI followed by counter staining with $4^{\prime}, 6$-diamidino-2-phenylindole (DAPI). Staining was performed by adding $50 \mu \mathrm{L}$ of PI solution in the dark. The stained buckypapers were then allowed to develop for c.a. $5 \mathrm{~min}$ before being rinsed with Milli-Q water. This process was then repeated for the counter stain, after which the buckypaper was again rinsed and stored in the dark prior to imaging using fluorescence microscopy.

Quantitative analysis of the fluorescent images was performed using the area-based estimation method outlined by Kang et al. [10]. This required each of the images obtained to be effectively split into two separate images showing the individual fluorescence attributable to each dye used to stain the buckypaper surface. These images were then converted to 8-bit greyscale images in which the colour intensity was converted into a 256 increment scale, with 0 corresponding to completely black and 255 to completely white. The software package used enabled the distribution of the brightness of pixels within the individual images to be determined. From the resulting curves, a threshold intensity value was chosen between 0 and 255 for all images, which allowed the subsequent production of a binary black and white image. In the latter, the white pixels were considered as representing the presence of fluorescence at a particular location on the buckypaper surface. Therefore by determining the ratio of black to white pixels in the image the percentage of the total buckypaper area that was fluorescing as a result of the presence of either live or dead bacteria could be calculated. By comparing these values for the two dyes used to stain each buckypaper, the percentage of dead bacteria could then 
TABLE 2: Physical properties of buckypapers.

\begin{tabular}{|c|c|c|c|c|c|c|}
\hline Buckypaper & Contact angle $\left({ }^{\circ}\right)$ & Conductivity $(\mathrm{S} / \mathrm{cm})$ & Young's modulus (GPa) & Tensile strength (MPa) & Ductility (\%) & Toughness $(\mathrm{J} / \mathrm{g})$ \\
\hline SWNT/Trix & $54 \pm 4$ & $85 \pm 2$ & $1.7 \pm 0.3$ & $20 \pm 10$ & $3.2 \pm 0.5$ & $0.3 \pm 0.2$ \\
\hline SWNT/cipro & $62 \pm 7$ & $70 \pm 20$ & $1.1 \pm 0.2$ & $7 \pm 2$ & $0.9 \pm 0.3$ & $0.05 \pm 0.02$ \\
\hline MWNT/Trix & $55 \pm 10$ & $24 \pm 16$ & $0.6 \pm 0.3$ & $6 \pm 3$ & $1.3 \pm 0.2$ & $0.10 \pm 0.06$ \\
\hline MWNT/cipro & $41 \pm 5$ & $42 \pm 1$ & $1.3 \pm 0.1$ & $6 \pm 2$ & $0.5 \pm 0.2$ & $0.04 \pm 0.01$ \\
\hline
\end{tabular}

TABLE 3: Removal of E. coli using buckypaper membranes.

\begin{tabular}{lc}
\hline Buckypaper & Log removal of E. coli \\
\hline SWNT/Trix & 2.96 \\
SWNT/cipro & 4.7 \\
MWNT/Trix & 5 \\
MWNT/cipro & $\mathrm{a}$ \\
$5 \mu \mathrm{m}$ PTFE membrane & 0.99 \\
\hline
\end{tabular}

${ }^{\mathrm{a}}$ Complete removal was observed for all membranes analyzed.

be determined. The previous experiments were performed in triplicate for SWNT/Trix and MWNT/Trix membranes, but only once, each for the corresponding buckypapers containing ciprofloxacin.

In addition to performing image analysis of buckypaper surfaces, the filtrates obtained after filtering solutions containing E. coli using either a SWNT/cipro or an MWNT/cipro membrane were stained and imaged to determine if any bacteria had crossed these buckypapers. In order to obtain images, a sample of the filtrate (c.a. $1 \mathrm{~mL}$ ) was centrifuged and the resulting pellet resuspended in sterile saline prior to casting onto a poly-L-lysine-coated microscope slide. The sample was then dried in air and stained with a combination of PI and DAPI as described previously.

\section{Results and Discussion}

3.1. Preparation and Characterisation of Dispersions Containing Ciprofloxacin. The ability of ciprofloxacin to disperse SWNTs has not been reported previously. Figure 2(a) shows the absorption spectrum of a $3 \mathrm{~mL}$ aqueous sample containing $0.1 \%(\mathrm{w} / \mathrm{v})$ SWNTs and $1.0 \%(\mathrm{w} / \mathrm{v})$ ciprofloxacin, which had been sonicated for different periods of time. A series of features related to the so-called van Hove singularities is apparent [24], and the absorbance at all wavelengths increased as the duration of sonication was lengthened. Both observations indicate that the SWNTs were becoming increasingly dispersed in the presence of the antibiotic. By plotting the absorbance of the solution at a given wavelength as a function of sonication time (e.g., Figure 2(b)), it can be seen that treatment for $30 \mathrm{~min}$ was sufficient to ensure significant dispersion of the SWNTs. Similar results to these were obtained when absorption spectra were recorded for a solution containing $0.1 \%(\mathrm{w} / \mathrm{v})$ MWNTs and $1.0 \%(\mathrm{w} / \mathrm{v})$ ciprofloxacin, although as expected the spectra lacked features attributable to the van Hove singularities.

3.2. Preparation and Characterisation of Buckypapers Containing Ciprofloxacin. Buckypapers were made by vacuum filtration of dispersions containing a total of either 30 or
$90 \mathrm{mg}$ of CNTs and either 1\% (w/v) cipro or 1\% (w/v) Trix. The thickness of all BPs was similar $(50 \mu \mathrm{m})$ regardless of their composition. Figure 3 presents SEM micrographs of the buckypapers, which reveal that their surface morphology varied depending on the dispersant and type of CNT used. Inspection of the micrographs also suggests that the diameter of the surface pores of the MWNT/Trix membrane are larger than that of the other buckypapers. This was further investigated by quantifying the diameter of the pores present on the surface of each of the four membranes using Image Pro Plus software. The results of this analysis are presented in Table 1 and confirm that the surface pores were the largest in the case of the MWNT/Trix membrane $(80 \pm 20 \mathrm{~nm})$. However, these were only slightly larger than those present on the surface of the MWNT/cipro buckypaper $(70 \pm 20 \mathrm{~nm})$. Inspection of the data in Table 1 also shows that the surface pores of both MWNT buckypapers are at least 2.5 times larger than those present for either SWNT membrane, suggesting that the choice of CNT is a major factor in determining surface morphology. This view is supported by an examination of the surface diameters of other SWNT buckypapers reported in our previously published study into the properties of a range of such materials. This included buckypapers synthesised from dispersions prepared using several low molecular mass dispersants including a cyclodextrin, a calixarene, a porphyrin, and a phthalocyanine [14]. For each of the latter materials analysis of the average surface pores evident in SEM images using Image Pro Plus software revealed that they were $<50 \mathrm{~nm}$, which is smaller than that of both MWNT buckypapers examined as part of the current study.

Further information about the surface area and average internal pore morphology of the BPs was obtained through analysis of nitrogen adsorption/desorption isotherms. Figure 4 shows the isotherms obtained for the SWNT/cipro, MWNT/cipro, and MWNT/Trix buckypapers. All may be categorized as general type IV isotherms that exhibit hysteresis at higher relative pressures. The isotherm for the SWNT/cipro buckypaper (Figure 4(a)) is very similar to those reported previously for other buckypapers prepared using the same type of SWNTs and low molecular mass dispersants including Trix [14]. In keeping with these previous results, the extent of nitrogen adsorption and desorption is significant at all relative pressures. In contrast, the isotherms obtained for the MWNT/cipro and MWNT/Trix buckypapers (Figures 4(b) and 4(c)) show that nitrogen adsorption and desorption occur predominantly at $P / P_{0}>0.8$. This suggests that there are significant differences between the internal morphologies of SWNT and MWNT buckypapers.

In order to investigate this hypothesis further, each of the isotherms in Figure 4 was subjected to analysis using 


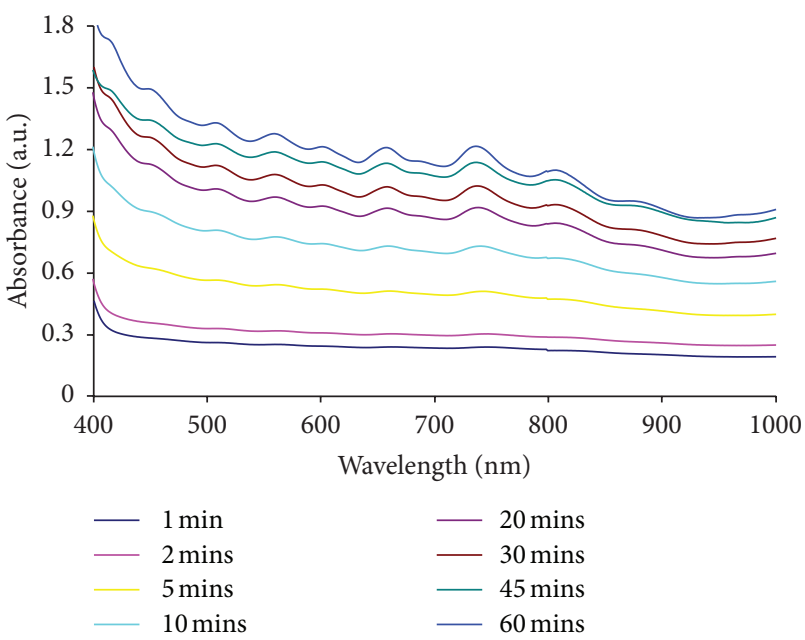

(a)

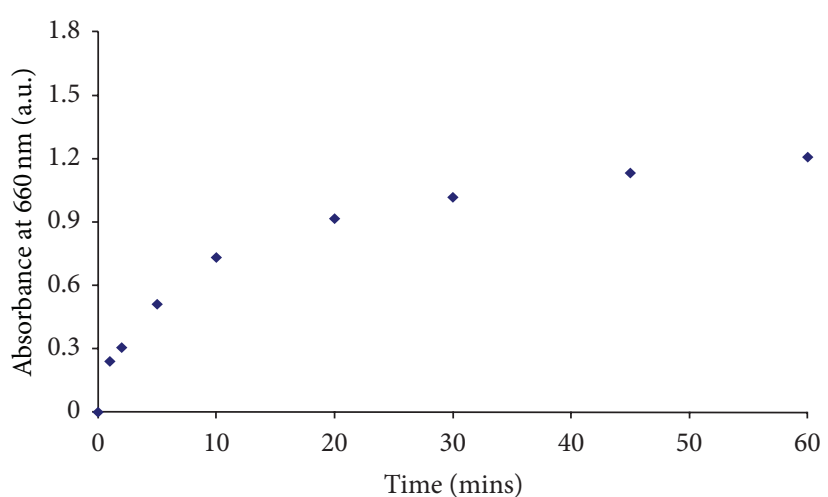

(b)

FIGURE 2: (a) Visible absorption spectra of a solution containing $0.1 \%(\mathrm{w} / \mathrm{v})$ SWNT and $1.0 \%$ (w/v) ciprofloxacin after different periods of sonication. (b) Effect of increasing sonication time on the absorbance at $660 \mathrm{~nm}$ of the previous SWNT/cipro dispersion.

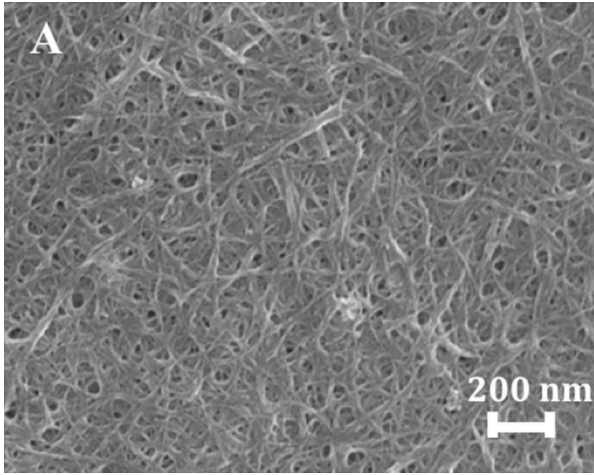

(a)

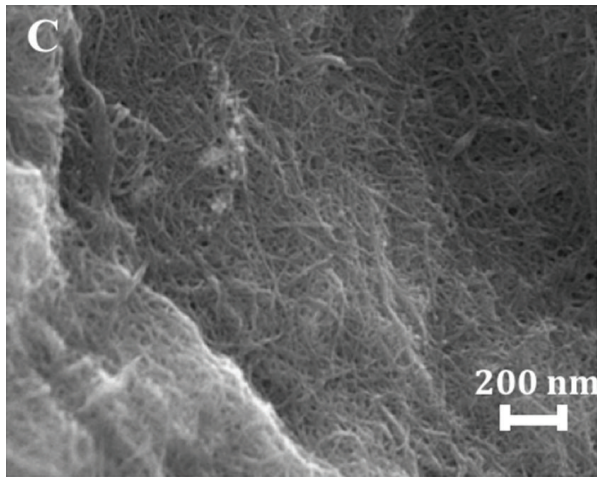

(c)

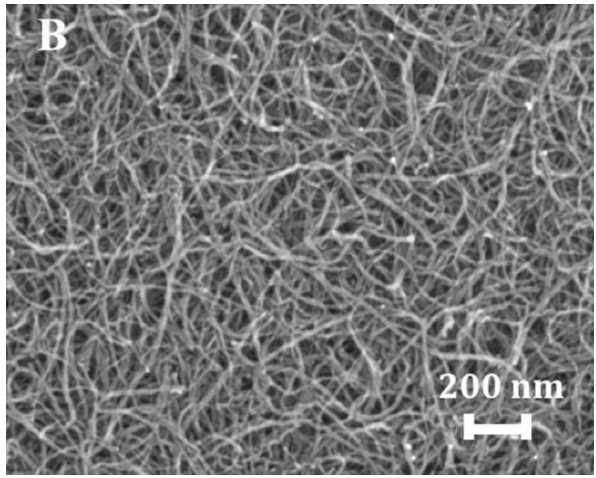

(b)

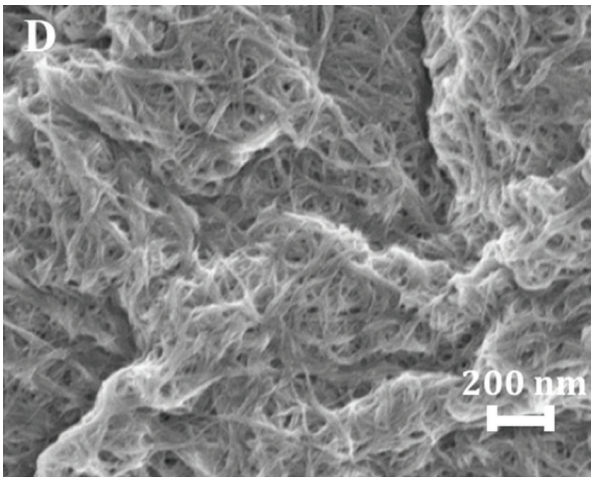

(d)

FIGURE 3: SEM micrographs (Mag 70,000x) of the surface of different buckypapers: (a) SWNT/Trix, (b) MWNT/Trix, (c) SWNT/cipro, and (d) MWNT/cipro.

the Barrett, Joyner and Halendar $(\mathrm{BJH})$ and HorvathKawazoe (HK) methods $[21,22]$. Analysis via the HK method afforded information on the distribution of small pores $(<2 \mathrm{~nm})$ within each of the membranes, while the $\mathrm{BJH}$ method allowed estimation of the larger pores. Combining the two sets of results yielded the pore size distribution profiles shown in Figure 5. Each set of curves show a large peak between 0.5 and $1.5 \mathrm{~nm}$, which can be attributed to the pores between individual nanotubes contained within CNT bundles (interstitial pores). In contrast, significant difference can be seen between the distributions of larger pores that occur between nanotube bundles for the SWNT and MWNT 


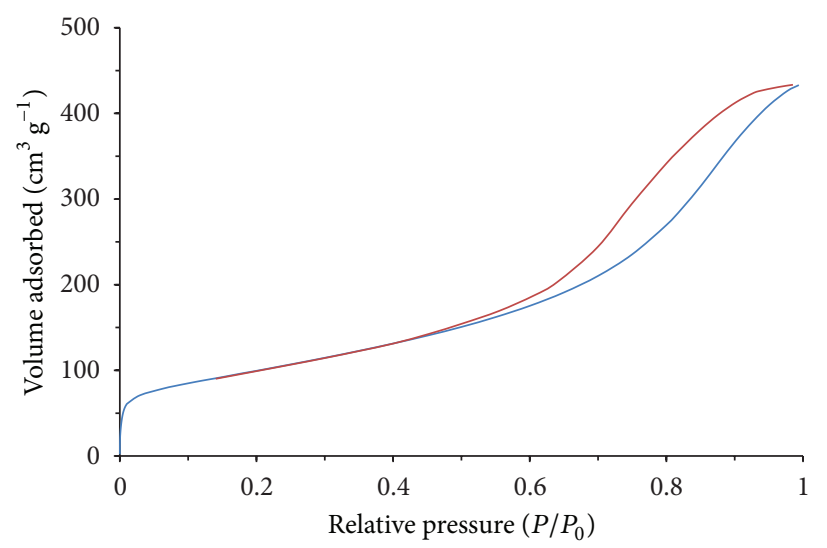

(a)

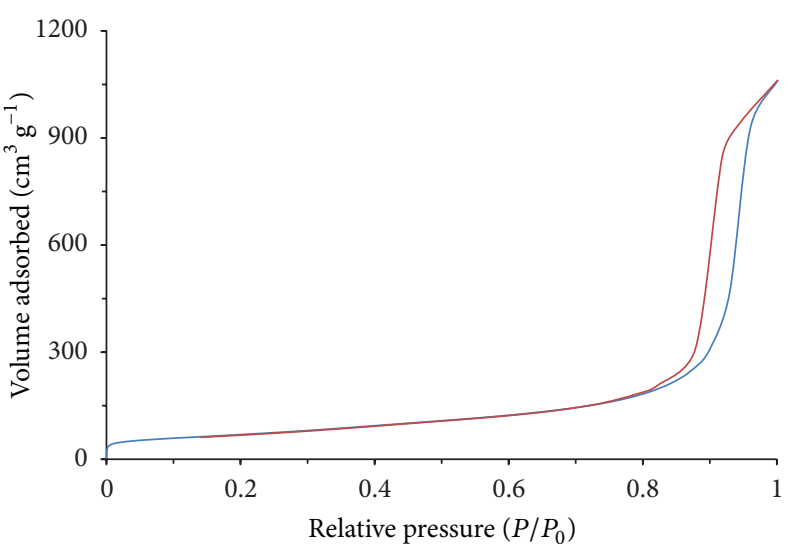

(b)

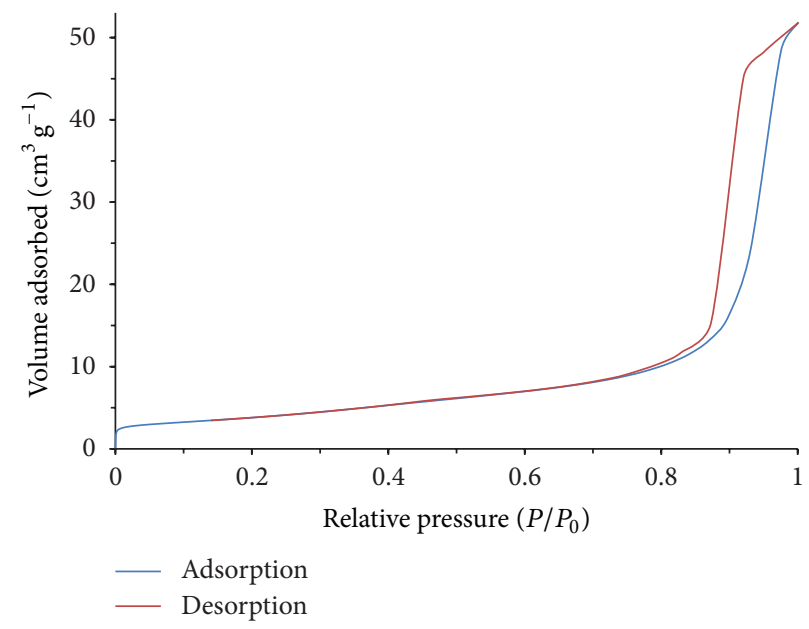

(c)

FIGURE 4: Nitrogen adsorption (blue)/desorption (red) isotherms for different buckypapers: (a) SWNT/cipro, (b) MWNT/cipro, and (c) MWNT/Trix.

buckypapers. In the case of the SWNT/cipro membrane (Figure 5(a)) a second, well-defined peak is centered at approximately $6 \mathrm{~nm}$ which can be attributed to the interbundle pores. For the two MWNT buckypapers, however, it is clear that the corresponding pores are significantly larger. In the case of the MWNT/Trix buckypaper (Figure 5(c)) the distribution of interbundle pores is centered at approximately $23 \mathrm{~nm}$. While there is no corresponding peak in the pore distribution curve for the MWNT/cipro buckypaper (Figure 5(b)), it is still clearly evident that the maxima in the peak distribution are located at $>15 \mathrm{~nm}$. Numerical integration of the sets of curves in Figure 5 was performed in order to calculate the average internal pore diameter of the membranes, as well as the percentage contribution of the interbundle pores to the total free volume. The results of this analysis, along with those obtained via application of the BET method [20] to the original isotherms, are presented in Table 1.

Of particular note is that the average diameter of the internal pores of the two SWNT membranes is significantly lower than that for the corresponding MWNT buckypapers, mirroring what was observed with the surface pores. Furthermore the average internal pore diameters obtained for the two SWNT membranes $(4 \pm 0.4$ and $7 \pm 0.8 \mathrm{~nm})$ are generally similar to values reported recently for other buckypapers prepared using the same batch of SWNTs and low molecular mass dispersants [14]. For example, the average internal pore diameter for a SWNT/Trix buckypaper was previously reported to be $4 \pm 0.4 \mathrm{~nm}$ [14]. However, it must be noted that one SWNT buckypaper in the latter study, that was prepared using phthalocyanine tetrasulfonic acid as the dispersant, was shown to possess internal pores with an average diameter of $27 \pm 3 \mathrm{~nm}$, which is comparable to that of the two MWNT membranes in the current study. In contrast to this, there was generally little difference between the average nanotube bundle diameter, internal pore volume, or surface area of SWNT and MWNT membranes in the current study. The one significant exception to this set of general trends was that the surface area of the SWNT/Trix buckypaper was more than two times bigger than that of any of the other materials.

Evidence that ciprofloxacin had been retained in the buckypapers was obtained by microanalysis. The atomic weight percentages of nitrogen and fluorine in a sample of a SWNT/cipro membrane were $2.8 \%$ and $1.2 \%$, respectively, while for a MWNT/cipro buckypaper these values were $2.0 \%$ 


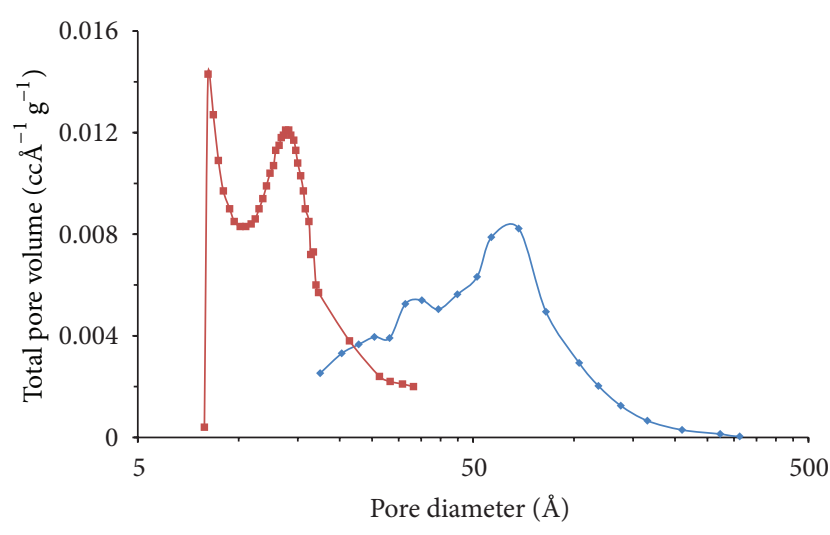

(a)

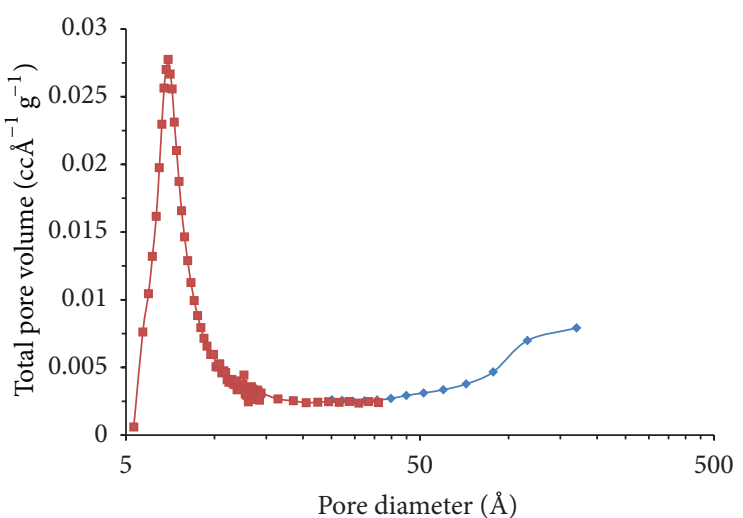

(b)

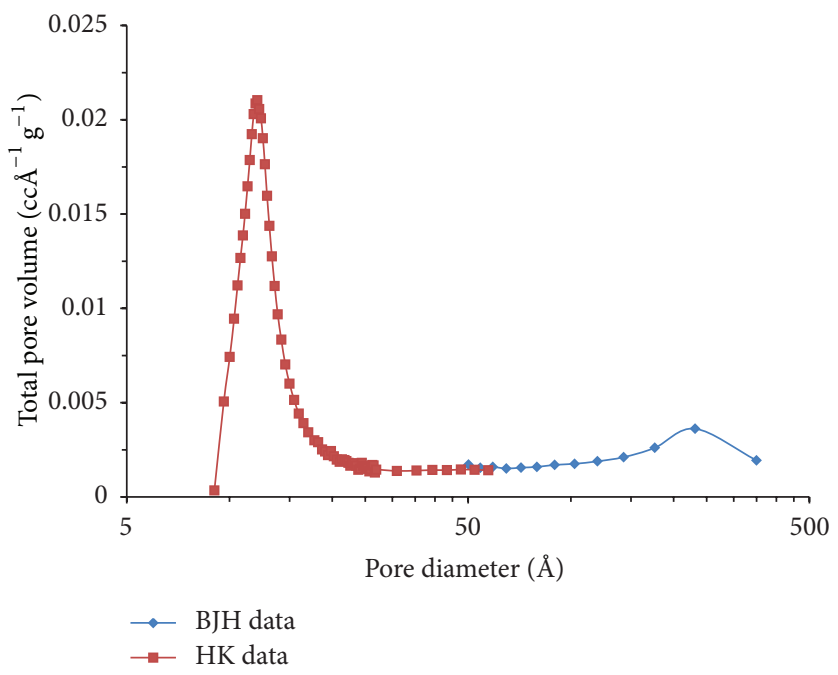

(c)

FIGURE 5: Pore size distributions for buckypapers derived by applying the HK method (red line) and BJH method (blue line) to data obtained from nitrogen adsorption/desorption isotherms: (a) SWNT/cipro, (b) MWNT/cipro, and (c) MWNT/Trix.

and $1.0 \%$, respectively. Both of these elements are present in ciprofloxacin but not in MWNTs or SWNTs. Further evidence in support of incorporation of antibiotic molecules in the buckypapers was obtained using energy dispersive Xray (EDX) spectroscopy. For example, the EDX spectrum (Figure 6(a)) of a MWNT/cipro membrane showed a peak with weak intensity at $\sim 0.65 \mathrm{keV}$ which is indicative of the presence of fluorine, and is absent from the corresponding spectrum of an MWNT/Trix buckypaper (Figure 6(b)).

The mechanical properties of the four buckypapers were investigated using a tensile test method, and the results obtained summarised in Table 2. Each of the mechanical properties determined from the stress-strain curves fell within a relatively narrow range of values. For example, the tensile strength of the materials was found to vary between $6 \pm 2$ and $20 \pm 10 \mathrm{MPa}$, while the Young's moduli were in the range $0.6 \pm 0.3-1.7 \pm 0.3 \mathrm{GPa}$. In all cases the mechanical properties of the buckypapers were found to be similar to those reported recently for other buckypapers prepared using the same batch of SWNTs and macrocyclic ligand dispersants [14]. Replacement of Trix by ciprofloxacin in both types of buckypapers generally resulted in a decrease in the mechanical properties of these materials. However, all buckypapers remained intact after being used for the bacterial filtration experiments described later, which typically lasted approximately for $1 \mathrm{~h}$. This suggests that they have sufficient mechanical integrity to allow their use for multiple filtration experiments. Each of the buckypapers containing Trix or cipro was found to be hydrophilic using the sessile drop method, which gave contact angles between $41 \pm 5$ and $62 \pm 7^{\circ}$. Measurement of the electrical conductivity of the buckypapers using a 2-point probe method gave values ranging from $24 \pm 16$ to $85 \pm 2 \mathrm{~S} \mathrm{~cm}^{-1}$, with the values for the materials prepared using SWNTs significantly larger than for those obtained using MWNTs. The conductivities obtained for the two SWNT buckypapers are comparable to those obtained for other membranes prepared using this class of CNTs and low molecular mass dispersants [14].

3.3. Bacterial Filtration Experiments. The ability of the buckypapers to remove E. coli JM109 was initially investigated by 


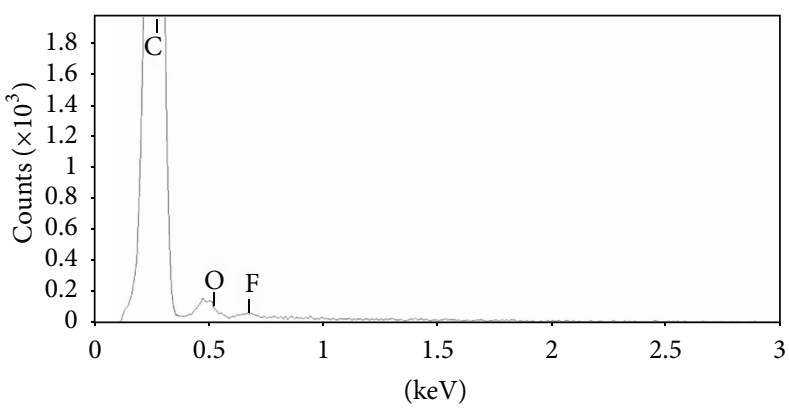

(a)

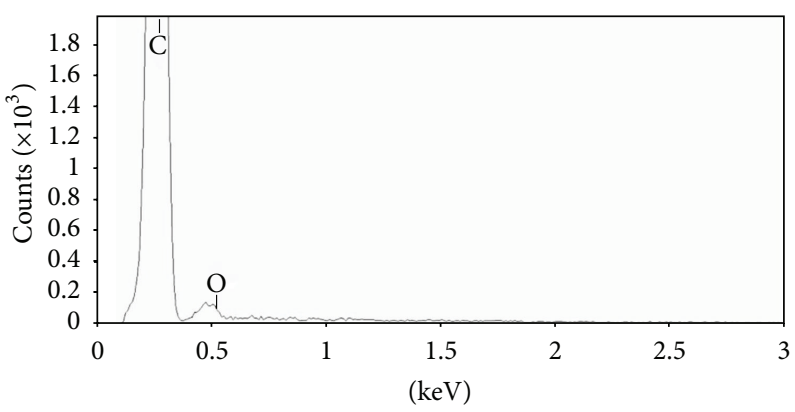

(b)

FIGURE 6: Energy Dispersive X-ray (EDX) Spectra of buckypapers: (a) MWNT/cipro and (b) MWNT/Trix.

vacuum filtering (at $200-300 \mathrm{mbar}$ ) $50 \mathrm{~mL}$ suspensions of the bacterium (in $0.9 \%(\mathrm{w} / \mathrm{v}) \mathrm{NaCl}$ ) with a final concentration of c.a. $10^{4}$ cells $\mathrm{mL}^{-1}$. Dilution series produced from the filtrates were plated onto Luria-Bertani (LB) agar and incubated overnight at $37^{\circ} \mathrm{C}$. Representative images of E. coli colonies grown from the initial bacterial suspension, and after filtering suspensions across either an MWNT/Trix or SWNT/Trix buckypaper, are shown in Figure 7. The total colony forming units (CFU) for each plate were counted and compared to the CFU for the initial bacterial suspension. The results of this analysis showed that each buckypaper removed $>99 \%$ of the E. coli present in the initial suspension, demonstrating that they were highly effective for this purpose. In contrast, when the $5 \mu \mathrm{m}$ PTFE membrane used as a support for preparing the buckypapers was used to filter the same E. coli suspension, only $90 \%$ of the bacteria were removed. In order to further facilitate comparison of the relative effectiveness of the different buckypapers, the percentage recoveries $(x)$ were converted into values of Log Removal using (1):

$$
\text { Log Removal }=-\log _{10}(100-x)+2 .
$$

Table 3 presents the values of Log Removal obtained for the various buckypapers, which suggest that buckypapers containing MWNTs were more effective for filtering E. coli than their SWNT counterparts containing the same dispersant. As anticipated, incorporation of ciprofloxacin instead of Trix into both types of buckypapers reduced the number of viable $E$. coli in the filtrates. In the case of experiments performed using MWNT/cipro buckypapers, complete removal of bacteria was observed for each of the three samples analysed, suggesting these membranes were the most effective for removal of E. coli.

Further evidence in support of this conclusion was provided by experiments in which the filtrates obtained using MWNT/cipro and SWNT/cipro buckypapers were stained using a combination of propidium iodide (PI) and DAPI and subsequently imaged using fluorescence microscopy. Propidium iodide is internalised only by membrane compromised (i.e., dead) bacterial cells and fluoresces red when excited with high-intensity light. In contrast, DAPI is able to enter all cells and fluoresces blue upon binding to DNA when appropriately excited with light. Figure 8 shows the fluorescence microscopic images obtained of the initial E. coli suspension as well as those of a filtrate obtained after filtering an identical sample of bacteria across a SWNT/cipro buckypaper. The image of the initial bacterial sample (Figure 8(a)) shows, as expected, blue regions attributable to the presence of viable E. coli cells, as well as red regions due to cells that had died as a result of natural attrition. In contrast, the image of the filtrate obtained using an SWNT/cipro buckypaper (Figure 8(b)) shows only a small number of red areas, indicating that some dead bacteria had passed across the membrane. The image of a filtrate obtained using an MWNT/cipro buckypaper did not show either red or blue regions, indicating that no bacterial cells passed across this membrane. This is consistent with the results presented previously that were obtained by counting the CFU, which indicated that the MWNT/cipro buckypaper was the most effective for filtering $E$. coli.

The general paucity of $E$. coli present in the filtrates obtained using each of the buckypapers can be attributed largely to rejection of bacteria owing to the greater size of their cells compared to the pores present on the surface and within the membranes and toxicity imparted onto the bacteria through contact with the nanotubes or dispersant molecules. The extent of inactivation of E. coli cells trapped on the surface of the buckypapers was assessed using a fluorescence-based viability assay reported previously [12]. In short, the surfaces of the four membranes were stained using a combination of either PI and SYTO-16 or PI and DAPI, after they had been used to filter the same number of bacterial cells and then imaged using fluorescence microscopy. Like DAPI, SYTO-16 is able to enter all cells. However, it fluoresces green instead of blue upon binding to DNA when excited with light of the appropriate wavelength.

Representative images of each of the four different types buckypapers, after they had been stained as described previously, are shown in Figure 9. Quantitative analysis of the images was performed in accordance with the method outlined by Kang et al. [10]. The results of analysis of the images presented in Figure 9 are presented in Table 4. Inspection of the data shows that there was perhaps a small difference in cell killing efficiency between the two buckypapers prepared using Trix as the dispersant, with the membrane synthesised using SWNTs appearing to be slightly more effective. Of more relevance is that the results show that both the MWNT and SWNT buckypapers displayed higher bactericidal properties when ciprofloxacin was present. For example, in the case of 


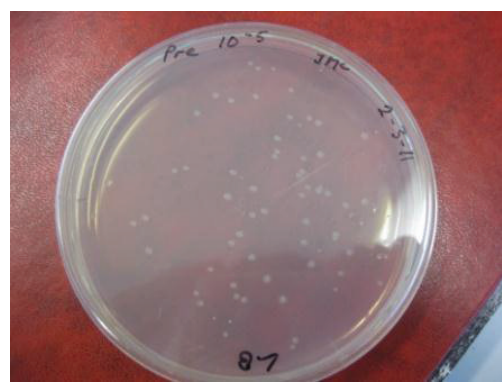

(a)

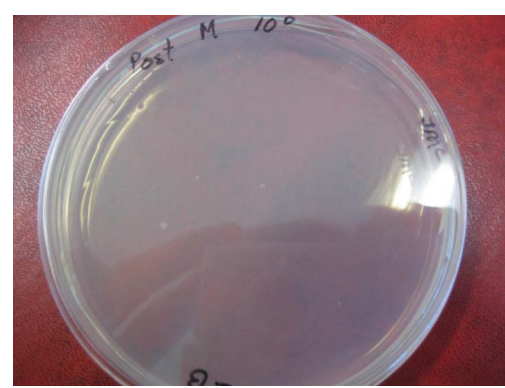

(b)

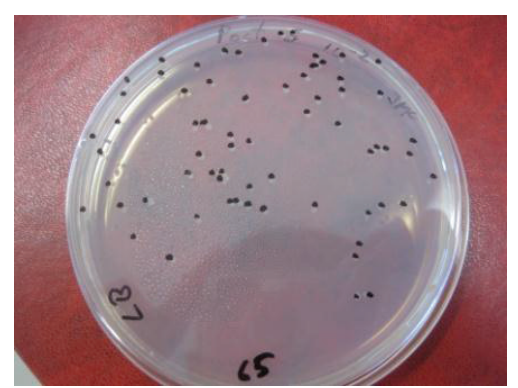

(c)

Figure 7: Images of LB plates after overnight culture of $100 \mu \mathrm{L}$ of (a) the initial E. coli suspension, (b) the filtrate obtained after passing the E. coli suspension across a MWNT/Trix membrane, and (c) the filtrate obtained after passing the E. coli suspension across a SWNT/Trix membrane. The initial E. coli suspension was diluted 100,000x before an aliquot was cultured. In the case of the filtrate obtained using an MWNT/Trix buckypaper, no dilution was performed before an aliquot was cultured, while the filtrate obtained using a SWNT/Trix buckypaper was diluted 100x before an aliquot was cultured. In the case of the image in (c) the positions of the cultures have been identified using a black marking pen in order to assist in counting the number of colonies present.

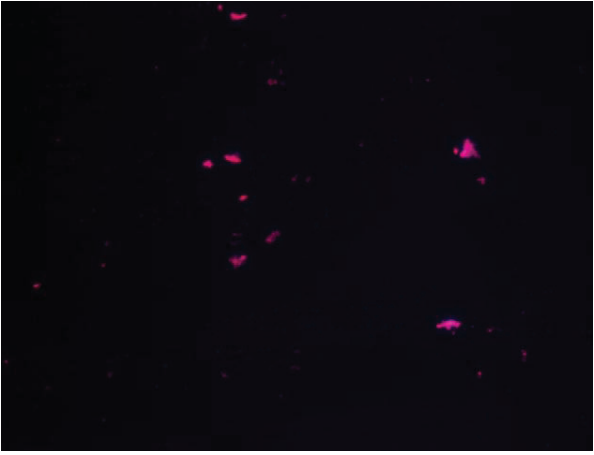

(a)

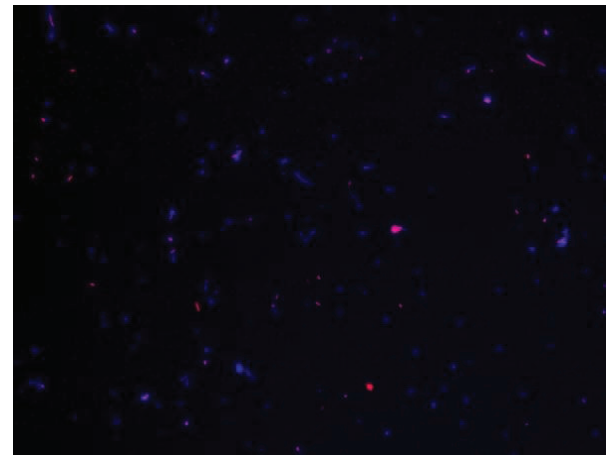

(b)

FIGURE 8: Fluorescence microscopic images of (a) an E. coli suspension in saline prior to a filtration experiment and (b) the filtrate obtained after passing an identical suspension of E. coli across a SWNT/cipro buckypaper. Both samples were stained with PI and DAPI.

MWNT buckypapers, the percentage of dead bacteria on the membrane surface increased from $58 \pm 13 \%$ for MWNT/Trix to $100 \%$ for MWNT/cipro.

\section{Conclusion}

In this paper we have demonstrated that ciprofloxacin can be used to assist the formation of dispersions of SWNTs. Furthermore buckypapers obtained from SWNT/cipro or MWNT/cipro dispersions retain antibiotic molecules after preparation. Analysis of SEM micrographs, and nitrogen adsorption/desorption isotherms, demonstrated that significant differences exist between the surface and internal morphologies of buckypapers prepared from MWNTs and SWNTs. In addition, the data also showed that replacing Trix as the dispersant used during buckypaper preparation by ciprofloxacin had little impact on these characteristics.

Each of the four buckypapers prepared removed more than $99 \%$ of the E. coli present in an aqueous suspension. This provides evidence that free-standing buckypaper membranes can be as effective for removing microbial contaminants from water supplies as the composite CNT materials investigated
TABLE 4: Bactericidal properties of buckypaper membranes.

\begin{tabular}{lc}
\hline Buckypaper & $\begin{array}{c}\text { Percentage of compromised bacteria on } \\
\text { buckypaper surface }\end{array}$ \\
\hline SWNT/Trix & $73 \pm 18$ \\
SWNT/cipro & $98^{\mathrm{a}}$ \\
MWNT/Trix & $58 \pm 13$ \\
MWNT/cipro & $100^{\mathrm{a}}$ \\
\hline
\end{tabular}

${ }^{\mathrm{a}}$ Values determined using one sample only.

previously $[12,13]$. It was somewhat surprising that the overall bacterial filtration efficiency of MWNT buckypapers prepared using either Trix or cipro was greater than that of the corresponding SWNT membranes. This suggests that there is something inherent in the structure of MWNT buckypapers that makes them more suitable for bacterial filtration applications.

Incorporation of ciprofloxacin significantly enhanced the ability of SWNT and MWNT buckypapers to impart bactericidal activity on E. coli. This demonstrates that it is possible to incorporate into buckypapers dispersant molecules with 


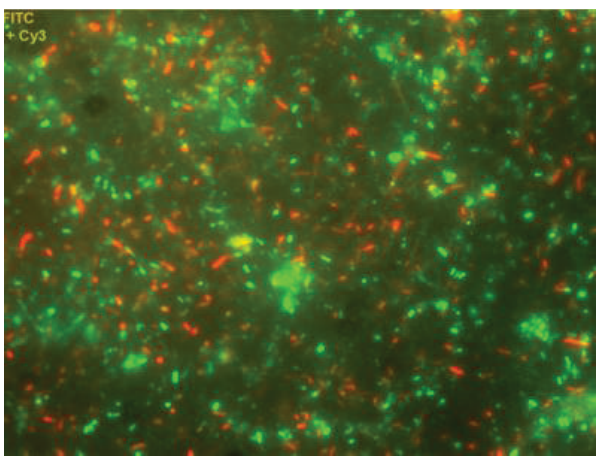

(a)

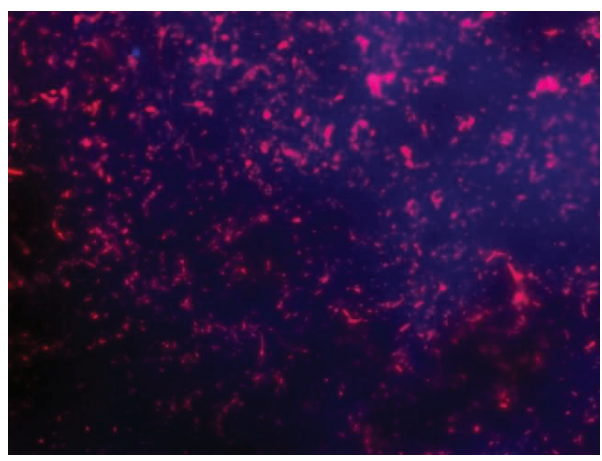

(c)

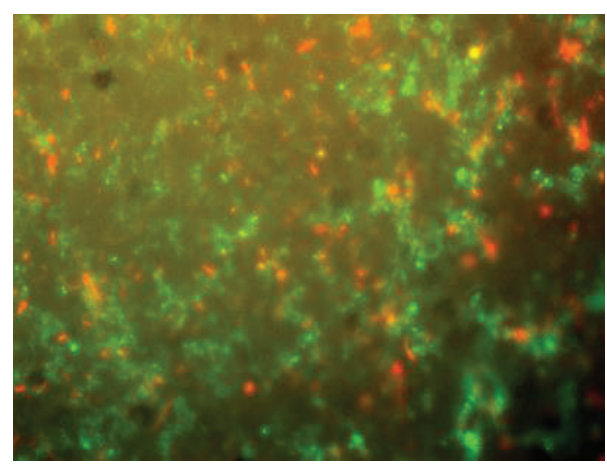

(b)

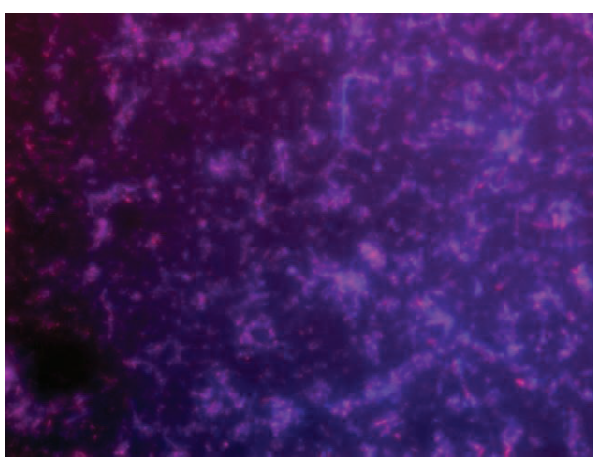

(d)

FIGURE 9: Fluorescence microscopic images of: (a) MWNT/Trix and (b) SWNT/Trix buckypapers stained with PI (red) and SYTO-16 (green) taken at 50x magnification and (c) MWNT/cipro and (d) SWNT/cipro buckypapers stained with PI (red) and DAPI (blue) taken at 20x magnification.

chemical and biological properties designed to improve their effectiveness for particular applications. This added functionality will only exist while the dispersant molecules are retained by the buckypaper. We therefore used absorption spectrophotmetry to monitor the leaching of ciprofloxacin from an MWNT/cipro buckypaper under conditions identical to those used for performing a bacterial filtration experiment. After one hour only $0.3 \mathrm{mg}$ of ciprofloxacin had leached from the buckypaper, which is less than $1 \%$ of its total mass. Future experiments are planned which will investigate what, if any, impact the loss of ciprofloxacin has on the efficacy of buckypapers when used multiple times for bacterial filtration experiments. In addition, we also intend to examine the effectiveness of our buckypapers for filtering solutions containing larger numbers of $E$. coli or different types of both gramnegative and gram-positive bacteria. We believe that these studies could provide insights into the reasons behind the observed difference in bacterial filtration efficiency between MWNT and SWNT membranes presented in this paper.

\section{Acknowledgments}

The authors acknowledge financial support from Australian Institute of Nuclear Science and Engineering (AINSE), Water Quality Research Australia, the New South Wales Environmental Trust, and the Australian Research Council (Centre of Excellence Funding and Future Fellowship to M. in het
Panhuis). The authors would like to thank Mr. Tony Romeo for the assistance obtaining SEM images and EDX spectra, Mrs Amanda Skora for the help performing the bacterial filtration experiments, and both Mr Nick Scales and Ms. Ilkay Chironi for the help performing nitrogen adsorption/desorption measurements. Mr Michael A. Ralph is also thanked for the assistance producing some of the images. The authors hereby declare that there is no conflict of interests resulting from the use of the chemicals or the equipment obtained from the nominated suppliers.

\section{References}

[1] J. P. S. Cabral, "Water microbiology. Bacterial pathogens and water," International Journal of Environmental Research and Public Health, vol. 7, no. 10, pp. 3657-3703, 2010.

[2] A. Fenwick, "Waterborne infectious diseases-could they be consigned to history?" Science, vol. 313, no. 5790, pp. 1077-1081, 2006.

[3] G. D. Kang and Y. M. Cao, "Development of antifouling reverse osmosis membranes for water treatment: a review," Water Research, vol. 46, no. 3, pp. 584-600, 2012.

[4] B. van der Bruggen, M. Mänttäri, and M. Nyström, "Drawbacks of applying nanofiltration and how to avoid them: a review," Separation and Purification Technology, vol. 63, no. 2, pp. 251$263,2008$.

[5] M. Ulbricht, "Advanced functional polymer membranes," Polymer, vol. 47, no. 7, pp. 2217-2262, 2006. 
[6] M. A. Shannon, P. W. Bohn, M. Elimelech, J. G. Georgiadis, B. J. Marĩas, and A. M. Mayes, "Science and technology for water purification in the coming decades," Nature, vol. 452, no. 7185, pp. 301-310, 2008.

[7] J. K. Holt, H. G. Park, Y. Wang et al., "Fast mass transport through sub-2-nanometer carbon nanotubes," Science, vol. 312, no. 5776, pp. 1034-1037, 2006.

[8] M. Majumder, N. Chopra, R. Andrews, and B. J. Hinds, "Nanoscale hydrodynamics: enhanced flow in carbon nanotubes," Nature, vol. 438, no. 7064, pp. 44-46, 2005.

[9] A. Srivastava, O. N. Srivastava, S. Talapatra, R. Vajtai, and P. M. Ajayan, "Carbon nanotube filters," Nature Materials, vol. 3, no. 9, pp. 610-614, 2004

[10] S. Kang, M. Pinault, L. D. Pfefferle, and M. Elimelech, "Singlewalled carbon nanotubes exhibit strong antimicrobial activity," Langmuir, vol. 23, no. 17, pp. 8670-8673, 2007.

[11] S. Kang, M. Herzberg, D. F. Rodrigues, and M. Elimelech, "Antibacterial effects of carbon nanotubes: size does matter!," Langmuir, vol. 24, no. 13, pp. 6409-6413, 2008.

[12] A. S. Brady-Estévez, S. Kang, and M. Elimelech, "A singlewalled-carbon-nanotube filter for removal of viral and bacterial pathogens," Small, vol. 4, no. 4, pp. 481-484, 2008.

[13] A. S. Brady-Estévez, M. H. Schnoor, S. Kang, and M. Elimelech, "SWNT-MWNT hybrid filter attains high viral removal and bacterial inactivation," Langmuir, vol. 26, no. 24, pp. 1915319158, 2010.

[14] L. J. Sweetman, L. Nghiem, I. Chironi, G. Triani, M. in het Panhuis, and S. F. Ralph, "Synthesis, properties and water permeability of SWNT buckypapers," Journal of Materials Chemistry, vol. 22, no. 27, pp. 13800-13810, 2012.

[15] L. Ji, W. Chen, S. Zheng, Z. Xu, and D. Zhu, "Adsorption of sulfonamide antibiotics to multiwalled carbon nanotubes," Langmuir, vol. 25, no. 19, pp. 11608-11613, 2009.

[16] L. Ji, W. Chen, L. Duan, and D. Zhu, "Mechanisms for strong adsorption of tetracycline to carbon nanotubes: a comparative study using activated carbon and graphite as adsorbents," Environmental Science and Technology, vol. 43, no. 7, pp. 23222327, 2009.

[17] S. A. Kumar, S. F. Wang, C. T. Yeh, H. C. Lu, J. C. Yang, and Y. T. Chang, "Direct electron transfer of cytochrome C and its electrocatalytic properties on multiwalled carbon nanotubes/ciprofloxacin films," Journal of Solid State Electrochemistry, vol. 14, no. 11, pp. 2129-2135, 2010.

[18] S. A. Kumar and S. F. Wang, "Adsorption of ciprofloxacin and its role for stabilizing multi-walled carbon nanotubes and characterization," Materials Letters, vol. 63, no. 21, pp. 18301833, 2009.

[19] S. A. Kumar, S. F. Wang, Y. T. Chang, H. C. Lu, and C. T. Yeh, "Electrochemical properties of myoglobin deposited on multiwalled carbon nanotube/ciprofloxacin film," Colloids and Surfaces B, vol. 82, no. 2, pp. 526-531, 2011.

[20] S. Brunauer, P. H. Emmett, and E. Teller, "Adsorption of gases in multimolecular layers," Journal of the American Chemical Society, vol. 60, no. 2, pp. 309-319, 1938.

[21] G. Horvath and K. Kawazoe, "Method for the calculation of effective pore size distribution in molecular sieve carbon," Journal of Chemical Engineering of Japan, vol. 16, no. 6, pp. 470475,1983 .

[22] E. P. Barrett, L. G. Joyner, and P. P. Halenda, "The determination of pore volume and area distributions in porous substances. I. Computations from nitrogen isotherms," Journal of the American Chemical Society, vol. 73, no. 1, pp. 373-380, 1951.
[23] F. M. Blighe, Y. R. Hernandez, W. J. Blau, and J. N. Coleman, "Observation of percolation-like scaling-far from the percolation threshold-in high volume fraction, high conductivity polymer-nanotube composite films," Advanced Materials, vol. 19, no. 24, pp. 4443-4447, 2007.

[24] H. Kataura, Y. Kumazawa, Y. Maniwa et al., "Optical properties of single-wall carbon nanotubes," Synthetic Metals, vol. 103, no. 1-3, pp. 2555-2558, 1999. 

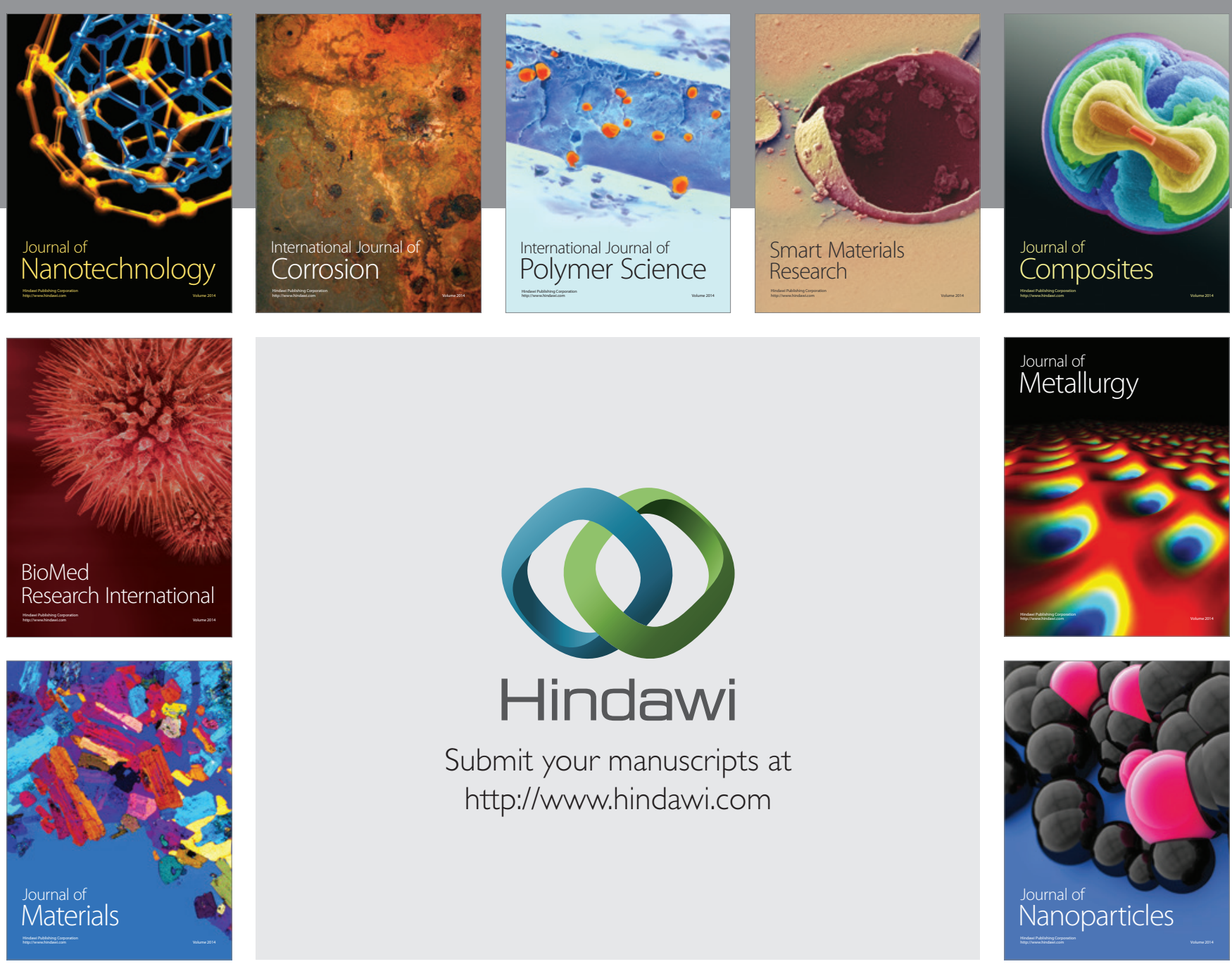

Submit your manuscripts at http://www.hindawi.com
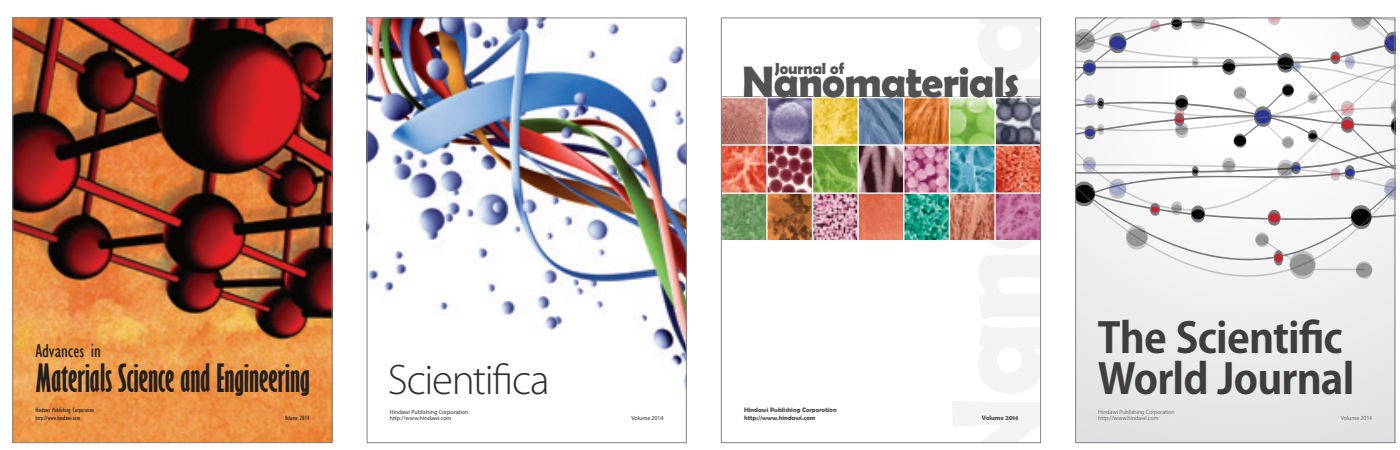

\section{The Scientific World Journal}
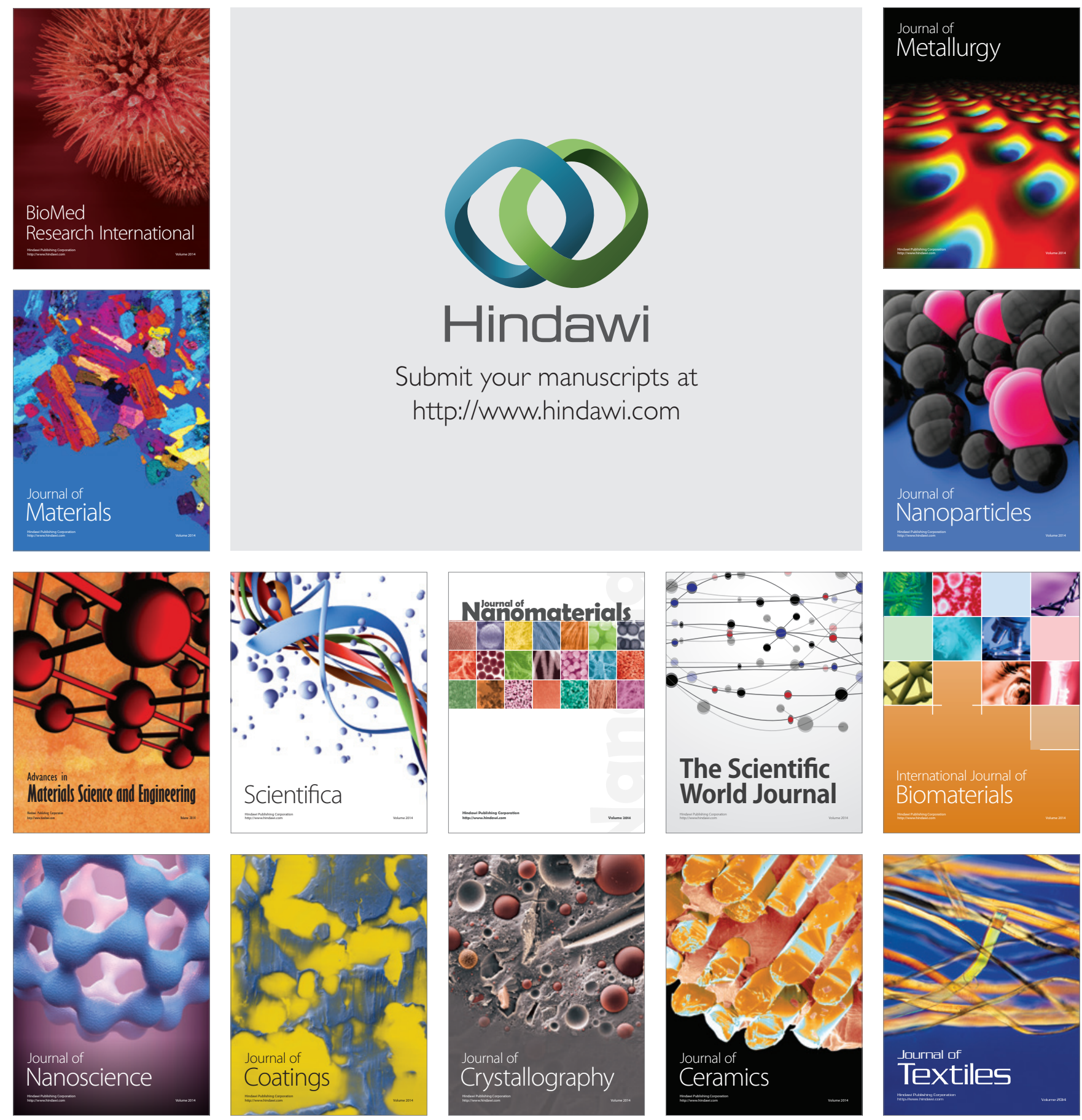